\title{
Validación de un instrumento para medir la calidad de vida en niños con mucositis orofaríngea en tratamiento por cáncer
}

\author{
Rosaura Gutiérrez-Vargas ${ }^{1}$, Eduardo Velasco-Rojano², Miguel Á. Villasís-Keever ${ }^{3}$, \\ Javier Portilla-Robertson ${ }^{4}$, Araceli Gutiérrez-Rodelo ${ }^{5}$, Sonny Flores-Navarro ${ }^{6}$, Luis E. Juárez-Villegas ${ }^{7}$ y \\ Marta Zapata-Tarrés ${ }^{8 *}$
}

${ }^{1}$ Programa de Maestría y Doctorado en Ciencias Médicas, Odontológicas y de la Salud, Universidad Nacional Autónoma de México, Ciudad de México; ${ }^{2}$ Universidad Nacional Autónoma de México, Ciudad de México; ${ }^{3}$ Unidad de Investigación en Epidemiología Clínica, Unidad Médica de Alta Especialidad Hospital de Pediatría, Centro Médico Nacional Siglo XXI, Instituto Mexicano del Seguro Social, Ciudad de México; ${ }^{4}$ Coordinación de Patología Bucal, División de Estudios de Posgrado, Facultad de Odontología, Universidad Nacional Autónoma de México, Ciudad de México; ${ }^{5}$ Servicio de Estomatología, Hospital Pediátrico de Sinaloa, Sinaloa; ${ }^{6}$ Servicio de Estomatología, Nuevo Hospital Civil de Guadalajara, Jalisco; ${ }^{7}$ Departamento de Oncología Médica, Hospital Infantil de México Federico Gómez, Ciudad de México; ${ }^{8}$ Departamento de Oncología Médica, Instituto Nacional de Pediatría, Secretaría de Salud, Cuidad de México. México

\begin{abstract}
Resumen
Introducción: La mucositis orofaríngea $(\mathrm{MO})$ es una de las principales complicaciones del tratamiento oncológico que reduce significativamente la calidad de vida (CV) del paciente. El objetivo fue traducir, adaptar de manera cultural y validar una nueva versión en español del instrumento Oropharyngeal Mucositis-Specific Quality-of-Life (OMQoL) en pacientes pediátricos. Métodos: Estudio transversal de validación, multicéntrico, realizado para la traducción y adaptación del OMQoL del inglés al español en pacientes de entre 8 y 16 años con MO. Se midió la confiabilidad mediante el Alfa de Cronbach; la validez del contenido y el constructo, con un análisis factorial exploratorio; y la validez convergente, con las correlaciones de las escalas para MO de la Organización Mundial de la Salud (OMS), la Oropharingeal Mucositis Assessment Scale (OMAS) y con el Pediatric Quality of Life-3 (PedsQL-3) módulo cáncer en español. Resultados: Participaron en el estudio 193 niños con una media de edad de $10.91 \pm 2.38$ años, de los cuales 101 (52.3\%) fueron de sexo femenino. En esta muestra, 80 niños (41.5\%) presentaron leucemia aguda linfoblástica y 111 (57.5\%) presentaron MO grado 2 y 3. El análisis factorial resultó con cuatro dimensiones con cargas > 0.40. De los 31 ítems del OMQoL, seis fueron eliminados. El Alfa de Cronbach del OMQoL español fue de 0.954. Las correlaciones de Spearman (r) con las escalas de la OMS y OMAS fueron significativas ( $r=-0.720$ y $r=-0.689 ; p<0.01$, respectivamente); con el PedsQL-3 módulo cáncer existió una moderada correlación ( $r=0.426 ; p<0.01)$. Conclusiones: La nueva versión del OMQoL en español demostró propiedades psicométricas adecuadas, y resulta un instrumento confiable y válido para medir la CV en niños con MO.
\end{abstract}

Palabras clave: Mucositis. Calidad de vida. Validación. Instrumentos. Niños.

Correspondencia:

*Marta Zapata Tarrés

E-mail: mzapatatarres@gmail.com
Disponible en internet: 11-01-2019 Bol Med Hosp Infant Mex. 2019;76:35-43

www.bmhim.com 


\title{
Validation of an instrument to measure the quality of life in children with oropharyngeal mucositis undergoing cancer treatment
}

\begin{abstract}
Background: Oropharyngeal mucositis (OM) is one of the primary complications arising during oncological treatment, which significantly reduces the patient's quality of life (QoL). The aim of this study was to translate, culturally adapt, and validate the use of a new Spanish version of the Oropharyngeal Mucositis-Specific Quality-of-Life instrument (OMQoL) for pediatric patients. Methods: A multicentric, cross-sectional validation study was conducted to translate and adapt OMQoL from English to Spanish for its use by children with OM aged 8-16 years. Reliability was measured using Cronbach's alpha; content and construct validity, in conjunction with exploratory factor analysis. The convergent validity, with the correlations of the scales for OM defined by the WHO, OMAS (Oropharingeal Mucositis Assessment Scale) and the PedsQL-3 cancer module in Spanish. Results: One hundred and ninety-three children with mean age of $10.91 \pm 2.38$ years participated in the study, out of which 101 (52.3\%) were females. In this sample, 80 children (41.5\%) suffered from acute lymphoblastic leukemia and $111(57.5 \%)$ had grade 2 and 3 OM. The factorial analysis resulted in four dimensions with loads $>0.40$. Among the 31 items of the OMQoL, six were eliminated. Cronbach alpha of OMQoL-Spanish was 0.954. Spearman's correlations $(r)$ with the OMS and OMAS scales were significant (with $r=-0.720$ and $r=-0.689 ; p<0.01$, respectively). Moderate correlation was observed with the PedsQL-3 cancer module $(r=0.426 ; p<0.01)$. Conclusions: OMQoL-Spanish demonstrated adequate psychometric properties, resulting in a reliable and valid instrument for measuring QoL in children with MO.
\end{abstract}

Key words: Mucositis. Quality of life. Validation. Instruments. Children.

\section{Introducción}

Los pacientes en tratamiento por cáncer padecen una serie de efectos celulares, principalmente en las células con alta tasa de replicación como las de la médula ósea, folículos pilosos y cavidad oral ${ }^{1}$. El daño directo de la terapia citotóxica hacia los tejidos orales produce una complicación frecuente denominada $m u$ cositis orofaríngea $(\mathrm{MO})^{2,3}$, que se manifiesta como eritema y edema en la mucosa hasta formar úlceras orales $^{4,5}$ (Fig. 1). La MO se presenta generalmente de 3 a 5 días después de iniciar la quimioterapia y alcanza su máxima intensidad entre los días 7 y 10, cerca del conteo más bajo de neutrófilos. En el caso de la radiación de cabeza y cuello se presenta aproximadamente al final de la primera semana ${ }^{6-8}$. Los pacientes de trasplante de células madre hematopoyéticas $(\mathrm{TCMH})$ refieren la MO como una de las complicaciones más dolorosas y debilitantes ${ }^{9}$. La morbilidad en pacientes por MO grave se asocia con dolor, a veces intratable y que necesita de analgesia narcótica, y con ingesta insuficiente de alimentos y líquidos que conlleva estados de desnutrición y deshidratación con requerimiento de nutrición parenteral, lo que da como resultado cambios psicológicos y el compromiso del tratamiento del cáncer, y que puede reducir significativamente la calidad de vida (CV) del paciente $5,9,10$. No todos los pacientes en tratamiento con quimioterapia desarrollan $\mathrm{MO}$, por lo que se han descrito factores de riesgo que pueden incrementar su desarrollo. Entre ellos destacan

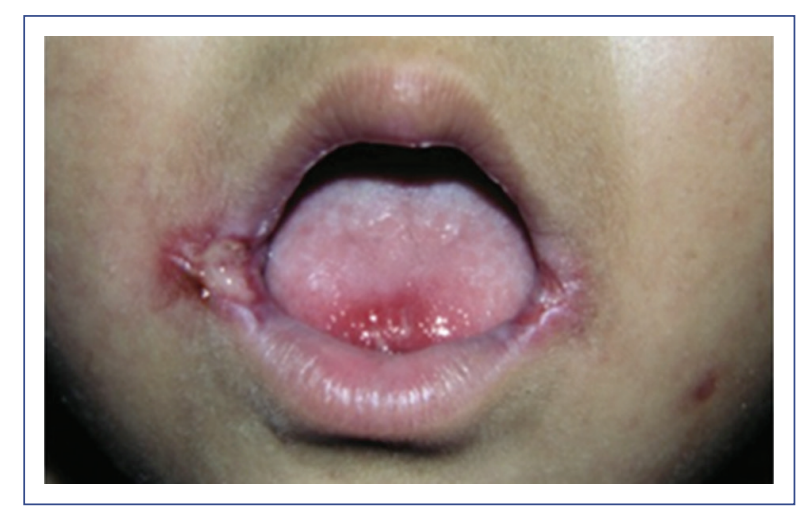

Figura 1. Paciente con mucositis orofaríngea grado 2 en las comisuras y la mucosa interna de los labios y la punta de la lengua.

la edad, debido a la velocidad de la mitosis epitelial ${ }^{9,11}$ y a la presencia de más receptores para el factor de crecimiento epidérmico en el epitelio ${ }^{8,12}$, el tipo de neoplasia hematológica, la condición oral pretratamiento, el grado de neutropenia y la desnutrición. La frecuencia de complicaciones orales se presenta hasta en el $85 \%$ de los pacientes con dosis intensas de quimioterapia y radioterapia ${ }^{13}$.

La CV relacionada con la salud se define como un constructo multidimensional que integra la percepción del paciente ante el impacto de la enfermedad y su tratamiento, así como su funcionamiento en diversos aspectos de la vida, incluyendo las áreas físicas, 
psicológicas y de la salud social ${ }^{14}$. La medición de la CV se basa en elementos subjetivos que requieren de instrumentos de evaluación válidos, reproducibles y confiables $^{15}$. Cuando son desarrollados en otros países con otro idioma o con el mismo, no pueden ser utilizados en nuestra población sin antes llevar un proceso de traducción, adaptación cultural y validación ${ }^{16}$.

Existen instrumentos para valorar la CV del paciente con MO que evalúan la gravedad de los síntomas, el dolor, los sentimientos, la limitación física. En conjunto, lo anterior interfiere con funciones de la vida diaria ${ }^{17}$, por lo que el instrumento Oropharyngeal MucositisSpecific Quality-of-Life (OMQoL) ${ }^{18}$ evalúa el impacto global de la MO en la CV desde la perspectiva de los pacientes. El propósito de este estudio fue traducir, adaptar culturalmente y validar el OMQoL en niños mexicanos en tratamiento por cáncer.

\section{Métodos}

Se trata de un estudio de validación, transversal, multicéntrico, realizado en cuatro hospitales pediátricos en México entre marzo del 2015 y julio del 2016. El tamaño de muestra calculado fue de acuerdo al coeficiente Alfa de Cronbach (poder: 0.80, $\alpha: 0.05$, consistencia interna: $0.70, \mathrm{~K}: 30)$ a través del programa PASS Sample Size 13. Se incluyeron pacientes de manera consecutiva de 8 a 16 años de edad, con diagnóstico de neoplasia hematológica o de tumor sólido, tratados con quimioterapia y radioterapia o trasplante de células madre hematopoyéticas (TCMH) con diversos grados de $\mathrm{MO}$ y que firmaron la carta de consentimiento y asentimiento informados. Se excluyeron aquellos pacientes cuyos padres, tutores o ellos mismos no tuvieran la capacidad para leer y contestar de manera autónoma el OMQoL. La investigación fue aprobada por los comités de ética e investigación de todas las instituciones participantes.

El OMQoL se obtuvo de manera directa de la Dra. Karis Cheng, autora original. El instrumento tiene 31 ítems que involucran cuatro dimensiones (sintomatología, alimentación, función social y sintomatología a la deglución) para estimar la CV. A cada ítem se le asigna una puntuación de 1 a 4 que describe desde "nada" a «mucho», con relación a si el encuestado ha tenido problemas durante los 3 días anteriores. Todas las puntuaciones se anotan de forma inversa (ejemplo, $1=4,2=3$, $3=2$ y $4=1$ ). Las puntuaciones de las subescalas se transforman linealmente a una escala de 0 a 100 ([total de las puntuaciones brutas de cada ítem de la subescala/puntaje bruto máximo posible de los ítems totales de la subescala] $x$ 100). El puntaje más alto indica una adecuada CV. EI OMQoL en inglés presenta una adecuada confiablidad (Alfa de Cronbach $>0.90$ ), validez convergente con la escala para MO de la Organización Mundial de la Salud (OMS) $(r=-0.724$ a $-0.971 ; p<0.01)$ y validez concurrente con la versión china del cuestionario de calidad de vida de la Organización Europea para la Investigación y Tratamiento del Cáncer, el European Organization for Research and Therapy of Cancer Quality of Life Questionnaire-30, Chinese version (EORTC QLQ-C30 [Ch]) $(r=0.450-0.724 ; p<0.01)^{18,19}$.

En este estudio, la MO fue evaluada con la escala para MO de la OMS ${ }^{2,20}$ y la Oral Mucositis Assessment Scale (OMAS), la cual mide el eritema (0: ninguno, 1: medio/moderado y 2: severo) y la ulceración o pseudomembrana (0: ninguno, $1:<1 \mathrm{~cm}^{2}, 2: 1-3 \mathrm{~cm}^{2}$ y 3 : $>3 \mathrm{~cm}^{2}$ ) en nueve sitios diferentes de la cavidad bu$\mathrm{cal}^{21}$. Las evaluaciones orales estuvieron a cargo de tres estomatólogas pediatras con previa estandarización interobservador (kappa $=0.72)$. Posteriormente, los participantes completaron el instrumento Pediatric Quality of Life-3 (PedsQL-3) modulo cáncer (versión de 8-12 y de 13-18 años de edad), adaptado y validado en población mexicana, el cual evalúa la CV de niños con cáncer tomando en cuenta signos y síntomas como dolor, náuseas, ansiedad ante procedimientos, ansiedad ante tratamientos, preocupaciones, problemas cognitivos y apariencia física. Los datos se convierten en los siguientes: $0 \rightarrow 100,1 \rightarrow 75,2 \rightarrow 50,3 \rightarrow 25$ y $4 \rightarrow 0$. El resultado es el promedio de la suma lineal de los ítems; el puntaje más alto refleja una adecuada $\mathrm{CV}^{22}$. Se incluyeron en el estudio solo los instrumentos con el $100 \%$ de ítems contestados.

Para el análisis estadístico se empleó el paquete estadístico SPSS versión 18 y estadística descriptiva para resumir las características demográficas y clínicas. La validez de contenido y constructo se estimó con el análisis factorial exploratorio. La correlación de Spearman entre el OMQoL y la escala de la OMS, OMAS y el PedsQL-3 módulo cáncer se calculó para estimar la validez convergente. La consistencia interna se calculó con el coeficiente Alfa de Cronbach.

\section{Resultados}

Se realizó la traducción directa del OMQoL al español por dos traductores independientes. Posteriormente, las dos versiones se unificaron, integrando una primera versión por medio de un grupo de expertos en medición y CV. Después se realizó la retrotraducción de esta versión en español al inglés por dos traductores diferentes. Se comparó la versión retrotraducida con la original y 
las diferencias se resolvieron mediante el consenso de expertos.

Se realizó una prueba piloto en 25 pacientes con una media de edad de $10.6 \pm 1.5$ años, con diferentes diagnósticos oncológicos y grados de $\mathrm{MO}$, con el objetivo de corroborar que los participantes comprendieran el nuevo instrumento. Antes de su aplicación, se dio una plática para mostrar las partes de la cavidad bucal y explicar términos clave como $\mathrm{MO}$ y úlceras. A los participantes se les dio a leer el nuevo OMQoL en español y se les pidió que hicieran sugerencias y comentarios con relación al instrumento. El tiempo aproximado de aplicación de la prueba fue de 15 minutos. Los participantes sugirieron modificar ciertos términos que para ellos no eran claros. Las palabras «hinchazón», «me angustia», «enrojecimiento», «sensación de ardor», «tengo dificultad», «nutritivo», «VOz más baja y no es clara», «bebo», «sólida» y «tragar» se modificaron a «hinchado», «me preocupa», «rojo», «me arde», «me cuesta trabajo», «sano», «hablo bajito y no me entienden», «tomo», «dura» y «pasarme la comida o saliva». Además, el enunciado «Tengo mi expresión (incluyendo el sonreír a los demás) y comunicación afectada» se cambió por «Mi cara, mi sonrisa y mi plática con los demás no es igual que antes». Debido a las sugerencias y modificaciones, se realizó una segunda prueba piloto en 18 pacientes con características similares, donde la mayoría de los enunciados fueron bien comprendidos. Se conformó la nueva versión del OMQoL en español adaptado para niños mexicanos. En las pruebas pilotos se observó que la mayoría de los niños tenían canalizada una vena en una o ambas manos, por lo que, en estos casos, los niños señalaban la respuesta y el investigador o los padres la circulaban.

Posteriormente, se incluyeron consecutivamente a 193 participantes, con una mediana de edad de 11 años (media $10.91 \pm 2.381$ y rango 8-16). El 52.3\% (101) fueron de sexo femenino, 63 (32.6\%) presentaron MO grado 2 y 48 (24.9\%), grado 3 (Fig. 1). Las características clínicas se describen en la tabla 1. Para obtener las características psicométricas del OMQoL en español se calcularon los estadísticos descriptivos para los 31 ítems (Tabla 2). Posteriormente, se probó el poder discriminativo de los ítems, es decir, su capacidad para detectar diferencias en las cantidades del atributo que se está midiendo, lo que, de acuerdo con Magnusson ${ }^{23}$, puede hacerse al probar que existe una relación sistemática entre los puntajes del ítem y el total de la prueba. Para esto se hizo una comparación de grupos extremos con respecto al total de la prueba (puntajes inferiores al primer cuartil y puntajes superiores al tercer cuartil)
Tabla 1. Características clínicas de los participantes del estudio de validación del OMQoL en español adaptado a niños

\begin{tabular}{|c|c|}
\hline Características & $\begin{array}{c}\text { Frecuencia (\%) } \\
\qquad n=193\end{array}$ \\
\hline $\begin{array}{l}\text { Diagnóstico } \\
\text { LAL Pre B } \\
\text { LAM } \\
\text { Osteosarcoma } \\
\text { Linfoma no Hodgkin } \\
\text { Rabdomiosarcoma alveolar } \\
\text { Sarcoma de Ewing } \\
\text { Rabdomiosarcoma embrionario } \\
\text { Linfoma linfoblástico de células T } \\
\text { LAL Pre T } \\
\text { Linfoma de Hodgkin } \\
\text { Schwannoma maligno } \\
\text { Otros }\end{array}$ & $\begin{array}{l}80(41.5) \\
26(13.5) \\
18(9.3) \\
9(4.7) \\
9(4.7) \\
8(4.1) \\
4(2.1) \\
4(2.1) \\
4(2.1) \\
3(1.6) \\
2(1.0) \\
26(13.4)\end{array}$ \\
\hline $\begin{array}{l}\text { Tratamiento oncológico } \\
\text { Quimioterapia } \\
\text { Quimioterapia/Radioterapia } \\
\text { TCMH } \\
\text { Radioterapia }\end{array}$ & $\begin{array}{c}176(91.2) \\
12(6.2) \\
3(1.6) \\
2(1.0)\end{array}$ \\
\hline $\begin{array}{l}\text { Etapa de tratamiento } \\
\text { Inducción } \\
\text { Consolidación } \\
\text { Mantenimiento } \\
\text { Primer ciclo } \\
\text { Segundo ciclo } \\
\text { Tercer ciclo } \\
\text { Cuarto ciclo } \\
\text { Reinducción }\end{array}$ & $\begin{array}{c}50(25.9) \\
35(18.2) \\
25(13) \\
17(8.8) \\
8(4.1) \\
7(3.8) \\
11(5.7) \\
10(5.2)\end{array}$ \\
\hline $\begin{array}{l}\text { Quimioterápicos } \\
\text { MTX } \\
\text { MTX-6MP } \\
\text { VCT-Doxo-CFM } \\
\text { MTX-L-aspar-Dexametasona } \\
\text { MTX-VP16-AraC } \\
\text { L-aspar } \\
\text { VCT-L-aspar-Dauno } \\
\text { IFM-Doxo } \\
\text { Cisplatino-Doxo } \\
\text { MTX-VCT } \\
\text { VCT } \\
\text { MTX-VP16-CFM } \\
\text { Etoposido-CFM } \\
\text { VCT- L-aspar } \\
\text { Otros }\end{array}$ & $\begin{array}{c}19(9.8) \\
13(6.7) \\
12(6.2) \\
11(5.7) \\
9(4.7) \\
8(4.1) \\
7(3.6) \\
6(3.1) \\
5(2.6) \\
5(2.6) \\
5(2.6) \\
4(2.1) \\
4(2.1) \\
4(2.1) \\
112(41.9)\end{array}$ \\
\hline $\begin{array}{l}\text { Grado de MO (OMS) } \\
\text { Grado } 0 \\
\text { Grado } 1 \\
\text { Grado } 2 \\
\text { Grado } 3 \\
\text { Grado } 4\end{array}$ & $\begin{array}{c}20(10.4) \\
44(22.8) \\
63(32.6) \\
48(24.9) \\
18(9.3)\end{array}$ \\
\hline Valor de OMAS & $\begin{array}{c}\text { Mediana } \\
0.60(0-2.5)\end{array}$ \\
\hline $\begin{array}{l}\text { Tipo de nutrición } \\
\text { Tolera la vía oral } \\
\text { Tolera únicamente líquidos } \\
\text { Parenteral } \\
\text { Enteral }\end{array}$ & $\begin{array}{c}127(65.8) \\
48(24.9) \\
13(6.7) \\
5(2.6)\end{array}$ \\
\hline
\end{tabular}


Tabla 1. Características clínicas de los participantes del estudio de validación del OMQoL en español adaptado a niños (Continuación)

\begin{tabular}{|l|c|}
\hline Características & $\begin{array}{c}\text { Frecuencia (\%) } \\
\mathbf{n = 1 9 3}\end{array}$ \\
\hline Tipo de analgésico & \\
Sin analgésico & $59(30.5)$ \\
Analgésico no opioide & $109(56.5)$ \\
Analgésico opioide & $25(13)$ \\
\hline
\end{tabular}

*Los datos se presentan como número, porcentaje, desviación estándar ( \pm ) y rango. AraC: citarabina; CFM: ciclofosfamida; Dauno: daunorrubicina; Doxo: doxorrubicina IFM: ifosfamida; LAL: leucemia aguda linfoblástica; LAM: leucemia aguda mieloblástica; L-aspar: L-asparginasa; MO: mucositis orofaríngea;

MTX: metotexato; OMAS: Oropharingeal Mucositis Assessment Scale:

OMS: Organización Mundial de la Salud; TCMH: trasplante de células madre hematopoyéticas; VCT: vincristina; VP16: etopósido; 6MP: 6 mercaptopurina.

con la prueba $U$ de Mann Whitney, misma que se seleccionó debido a que la distribución de los ítems no fue normal. Todos los ítems fueron conservados por discriminar significativamente.

Con estos datos se realizó un análisis de consistencia interna, y se obtuvo un Alfa de Cronbach de 0.963, y un análisis de correlación entre los ítems y el total de la suma de los mismos para identificar el tipo de método de extracción de factores y el método de rotación necesaria para los datos obtenidos. Con esta prueba se observó que los datos presentaban correlaciones de medias a altas ( $\geq 0.60)$, por lo que se eligió el método de extracción de factores de ejes principales y el método de rotación oblicua Promax ${ }^{24}$.

Los resultados mostraron un valor de adecuación de la muestra Kaiser-Meyer-Olkin de 0.952 y una prueba de esfericidad de Barlett significativa ( $p \leq 0.001$ ), lo que indica un excelente grado de relación conjunta de las variables, y ello es apropiado para realizar un análisis factorial (Tabla 3). Se eliminaron dos ítems (18 y 21 con cargas factoriales de 1.003 y 1.000 , respectivamente) por ser casos Heywood (dado que la solución no es interpretable por tener comunalidades $\geq 1$ y debido a la imposibilidad de tener más del $100 \%$ de varianza) ${ }^{24}$. De forma similar, se eliminaron los ítems 10, 12,15 y 19 de la dimensión dieta por presentar cargas factoriales $<0.40$ en los cuatro factores ${ }^{25}$.

El análisis factorial exploratorio indicó que la escala se compone de cuatro factores que explican el $56.672 \%$ de la varianza con 25 ítems (validez de constructo). Nuevamente se calculó la confiablidad de la nueva versión en español del OMQoL, y se obtuvo un Alfa de Cronbach de 0.954 (IC 95\%: 0.941-0.972) para el total de la escala. La correlación entre el OMQoL en español y la escala de la OMS para MO fue significativa $(r=-0.720 ; p<0.01)$, al igual que con la escala OMAS $(r=-0.689 ; p<0.01)$. La correlación con el PedsQL-3 módulo cáncer para niños fue moderada pero significativa $(r=0.426 ; p<0.01)$, y resultó en una adecuada validez convergente (Tabla 4 ).

\section{Discusión}

El enfoque que involucra la relación de CV y MO está en constante crecimiento como un área de evaluación integral de la terapia oncológica. Sin embargo, la información en niños es limitada porque no se cuenta con instrumentos específicos para este grupo de edad. Debido a esto, en ocasiones se emplean instrumentos genéricos y enfocados a adultos para estimarla. Ejemplos de estos instrumentos son el Oral Health Impact Profile-14 (Perfil de Impacto de la Salud Oral-14), que omite signos y síntomas, como sangrado, ardor bucal, percepción de la saliva, fonación, deglución, disgeusia y dolor de garganta, que frecuentemente refieren los pacientes con $\mathrm{MO}^{26,27}$; el Functional Assessment of Cancer Therapy-Head and Neck (Evaluación Funcional de la Terapia del Cáncer - Cuestionario cabeza y cueIlo) ${ }^{28}$; y el European Organization for Research and Therapy of Cancer-Quality of Life Questionnaire-30 (Instrumento de la Organización Europea para la Investigación y Tratamiento del Cáncer-Calidad de Vida Cuestionario 30$)^{29}$. Todos ellos tienen una utilidad limitada para la extensa sintomatología que involucra la MO. Otros, como el Patient-Reported Oral Mucositis Symptoms $^{30}$ y el Oral Mucositis Weekly Questionnaire-Head and Neck Cancer ${ }^{31}$, miden los síntomas de $\mathrm{MO}$ - excluyendo toda una gama de características que la rodean-y no incluyen elementos para evaluar la CV.

En el presente estudio, 80 pacientes (41.5\%) padecían leucemia aguda linfoblástica, la neoplasia maligna que afecta alrededor del $50 \%$ de los niños con cáncer, seguida de la leucemia mieloide y el linfoma. Los pacientes con tumores sólidos principalmente presentaron osteosarcoma, ya que es el tipo de tumor que más afecta a los adolescentes. Estas enfermedades se tratan con protocolos intensos que producen mielosupresión y daño a las mucosas, por lo que influyen en la proporción de los distintos grados de $\mathrm{MO}$ : el grupo con grado 4 fue el que presentó el menor porcentaje (9.3\%). La evaluación psicométrica de la nueva versión del OMQoL en español reveló que los ítems eran representativos de la CV y MO al presentar una adecuada validez convergente, a pesar de existir adecuaciones para su traducción en español y adaptación a niños. El análisis factorial mostró un nuevo orden en las 
Tabla 2. Resultados del análisis de los reactivos del OMQoL-español adaptado a niños

\section{Reactivos}

1. Tengo hinchado dentro de mi boca

2. Tengo úlceras en mi boca

3. El dolor en la boca me preocupa

4. Tengo pus o sangre en mis labios o dentro de mi boca

5. Siento molestia al cepillarme los dientes o enjuagarme la boca

6. El dolor en la boca no me deja dormir

7. Me duele la boca

8. Tengo rojo o me arde dentro de mi boca

9. Me cuesta trabajo abrir mi boca

10. No puedo disfrutar la comida

11. No me gusta comer fuera de mi casa por la molestia en la boca

12. Mi saliva se vuelve pesada o pegajosa y necesito escupir muchas veces

13. Tengo cambios en los sabores

14. La dificultad para comer me preocupa

15. Me tardo más tiempo en comer o beber

16. He bajado de peso

17. He cambiado mi comida

18. Como menos alimentos suaves y duros

19. Me preocupa que coma poco y no sea sano

20. Hablo con voz bajita y no me entienden

21. Tengo dificultad para hablar

22. Necesito usar otras cosas (por ejemplo papel y pluma o señas para hablar con los demás)

23. Me da vergüenza comer con mi familia o amigos

24. La dificultad para hablar me preocupa

25. No quiero hablar con otras personas (ni por teléfono) porque me molesta mi boca

26. Mi cara, mi sonrisa y mi comunicación con los demás no son igual que antes

27. Me duele la garganta

28. Tomo pocos líquidos (por ejemplo, agua, jugo o sopa)

29. Me cuesta trabajo pasarme la comida blanda y dura

30. Siento que me ahogo fácilmente al pasar los líquidos, la comida y la saliva

31. Me cuesta trabajo pasarme la saliva

OMQoL: Oropharyngeal Mucositis-Specific Quality-of-Life.
Media Desviación estándar $\quad$ Sesgo

\begin{tabular}{l|l|l}
2.98 & 1.005 & -0.69
\end{tabular}

2.91

2.99

3.46

0.936

$-0.616$

1.005

$-0.727$

2.92

0.866

1.550

2.92

1.012

$-0.942$

3.44

0.934

1.231

2.59

2.73

1.072

$-1.235$

3.05

0.980

$-1.030$

2.93

1.067

$-0.829$

2.90

1.120

$-1.195$

2.90

1.236

$-1.390$

1.139

$-1.270$

3.01

0.976

$-0.674$

2.94

1.059

$-0.992$

2.67

1.096

$-1.284$

2.90

1.023

$-0.994$

2.68

1.090

$-1.290$

2.67

2.90

1.082

$-1.268$

3.08

1.109

$-1.126$

3.52

1.082

$-0.827$

3.52

1.092

$-0.799$

3.52

0.884

1.586

3.48

$$
0.896
$$

1.720

3.22

1.014

$-0.209$

3.17

2.95

1.079

$-0.600$

1.107

$-1.045$

3.13

3.21

1.052

$-0.856$

0.990

$-0.226$

2.98

1.082

$-1.028$

3.49

0.879

1.383

3.24

0.983

$-0.656$ dimensiones: la subescala de función social se posicionó como la primera dimensión (nueve ítems), seguido de síntomas (siete ítems), deglución (seis ítems) y dieta (tres ítems). Con ello pudo explicarse por qué, en nuestra cultura, la expresión y la comunicación son muy importantes, seguido de la evidente afección que produce la MO. A pesar de la eliminación de cinco ítems en la subescala de dieta («No puedo disfrutar la 
Tabla 3. Cargas factoriales de la matriz de componentes rotados del OMQoL-español adaptado a niños

\begin{tabular}{|c|c|c|c|c|}
\hline \multirow[t]{3}{*}{ Elementos } & \multicolumn{4}{|c|}{ Cargas factoriales } \\
\hline & \multicolumn{4}{|c|}{ Factor } \\
\hline & 1 Función social & 2 Síntomas & 3 Deglución & 4 Dieta \\
\hline $\begin{array}{l}\text { 25. No quiero hablar con otras personas (ni por teléfono) porque } \\
\text { me molesta la boca }\end{array}$ & 0.947 & -0.042 & -0.044 & 0.083 \\
\hline 24. La dificultad para hablar me preocupa & 0.898 & -0.005 & 0.008 & -0.023 \\
\hline 20. Hablo con voz más bajita y no me entienden & 0.847 & -0.164 & 0.076 & 0.108 \\
\hline $\begin{array}{l}\text { 22. Necesito usar otras cosas (por ejemplo papel y pluma o señas) } \\
\text { para poder comunicarme con los demás }\end{array}$ & 0.844 & -0.073 & 0.017 & 0.033 \\
\hline 23 Me da vergüenza comer con mi familia o amigos & 0.694 & 0.068 & 0.094 & -0.300 \\
\hline 9. Me cuesta trabajo abrir mi boca & 0.473 & 0.378 & -0.166 & 0.157 \\
\hline 14. La dificultad para comer me preocupa & 0.470 & 0.370 & -0.060 & 0.132 \\
\hline $\begin{array}{l}\text { 11. No me gusta comer fuera de mi casa por las molestias en la } \\
\text { boca }\end{array}$ & 0.427 & 0.226 & 0.077 & 0.020 \\
\hline $\begin{array}{l}\text { 26. Mi cara, mi sonrisa y mi comunicación con los demás no son } \\
\text { igual que antes }\end{array}$ & 0.423 & 0.1940 & 0.065 & 0.207 \\
\hline 5. Siento molestia al cepillarme los dientes o enjuagarme la boca & -0.187 & 0.848 & -0.053 & 0.046 \\
\hline 2. Tengo úlceras en mi boca & 0.148 & 0.695 & -0.104 & 0.099 \\
\hline 3. El dolor en la boca me preocupa & -0.009 & 0.672 & 0.100 & -0.009 \\
\hline 1. Tengo hinchado dentro de mi boca & 0.040 & 0.566 & 0.162 & -0.012 \\
\hline 8. Tengo rojo o sensación de ardor dentro de mi boca & 0.035 & 0.499 & 0.078 & 0.199 \\
\hline 4. Tengo pus o sangre en mis labios o dentro de mi boca & 0.188 & 0.447 & 0.144 & -0.292 \\
\hline 6. El dolor en la boca no me deja dormir & 0.087 & 0.422 & 0.275 & -0.110 \\
\hline 27. Me duele mi garganta & -0.037 & 0.008 & 0.788 & 0.067 \\
\hline 31. Me cuesta trabajo tragarme la saliva & 0.011 & 0.003 & 0.786 & 0.118 \\
\hline $\begin{array}{l}\text { 30. Siento que me ahogo fácilmente al pasar los líquidos, la comida } \\
\text { y la saliva }\end{array}$ & 0.068 & 0.159 & 0.703 & -0.079 \\
\hline 28. Tomo poquitos líquidos (por ejemplo, agua, jugo o sopa) & 0.168 & 0.014 & 0.509 & -0.004 \\
\hline 13. Tengo cambios en los sabores & -0.124 & -0.134 & 0.453 & 0.371 \\
\hline 29. Me cuesta trabajo pasarme la comida (blanda y dura) & 0.228 & 0.131 & 0.451 & 0.086 \\
\hline 7. Me duele la boca & -0.132 & 0.140 & -0.020 & 0.615 \\
\hline 16. He bajado de peso & 0.130 & -0.220 & 0.155 & 0.545 \\
\hline $\begin{array}{l}\text { 17. He cambiado mi comida (por ejemplo, el tipo de alimentos, } \\
\text { forma y tamaño) }\end{array}$ & 0.011 & 0.252 & 0.077 & 0.543 \\
\hline
\end{tabular}

OMQoL: Oropharyngeal Mucositis-Specific Quality-of-Life.

comida", "Mi saliva se vuelve pesada o pegajosa y necesito escupir muchas veces-, «Me tardo más tiempo en comer o beber», "Me preocupa que coma poco y no sea sano", "Como menos alimentos suaves y duros» y "Tengo dificultad para hablar») y uno de función social («Tengo dificultad para hablar»), el instrumento conservó una adecuada confiabilidad (Alfa de Cronbach $=0.954)$. 
Tabla 4. Correlación del OMQoL en español con el PedsQL-3 módulo cáncer, Escala de MO (OMS) y Escala OMAS*

\begin{tabular}{|c|c|}
\hline & OMOoL en español adaptado a niños, valor de $p$ \\
\hline Escala para MO según la OMS (grado de severidad de MO) & $-0.735^{* *}$ \\
\hline Escala OMAS & $-0.690^{* *}$ \\
\hline PedsQL-3 Módulo cáncer (8-12 años/13-18 años) & $0.426^{* *}$ \\
\hline
\end{tabular}

Cuando se pretende adaptar culturalmente un instrumento a un país con idioma diferente al original $y$, en este caso, a una edad diferente, es probable que algunos ítems no sean bien comprendidos o sean irrelevantes. Esto se ve reflejado en cargas factoriales bajas y la consecuente necesidad de eliminación de ítems, ya que conservarlos podría conducir a un sesgo de información e interpretación por parte de los participantes ${ }^{16}$.

Las correlaciones del OMQoL con las escalas de MO (OMS y OMAS) mostraron una adecuada validez convergente, mientras que la correlación con el PedsQL-3 fue moderada. Posiblemente, esto se debe a que el PedsQL-3 evalúa más áreas de la vida, que, aunque pudieran relacionarse con $\mathrm{MO}$, no se centran en el mismo criterio de evaluación, sino que involucran una serie de variables a las cuales están expuestos los pacientes oncológicos, como el dolor corporal, el dolor y temor a las canalizaciones o aspirados de medula ósea, las náuseas, el temor al médico u hospital, la preocupación por los efectos secundarios de su tratamiento y la afectación en la apariencia física.

La evaluación de la CV en pacientes con MO debe realizarse con instrumentos específicos, confiables y válidos para esta relación, ya que los instrumentos generales no pueden captar los signos y síntomas orofaríngeos que pueden llegar a limitar la alimentación, comunicación y apariencia física percibida, entre otros. Esto se presenta sobre todo en niños que, por su corta edad, no comprenden el panorama de su diagnóstico y tratamiento, además del dolor en la boca que presentan — secundario a las úlceras-, el impacto del sangrado bucal y la incapacidad de abrir la boca o comunicarse, lo cual implica para ellos una gran angustia y desesperación.

\section{Responsabilidades éticas}

Protección de personas y animales. Los autores declaran que para esta investigación no se han realizado experimentos en seres humanos ni en animales.
Confidencialidad de los datos. Los autores declaran que han seguido los protocolos de su centro de trabajo sobre la publicación de datos de pacientes.

Derecho a la privacidad y consentimiento informado. Los autores han obtenido el consentimiento informado de los pacientes y sujetos referidos en el artículo. Este documento obra en poder del autor de correspondencia.

\section{Conflicto de intereses}

Los autores declaran no tener ningún conflicto de intereses.

\section{Financiamiento}

Esta investigación no contó con financiamiento.

\section{Agradecimientos}

Nuestro agradecimiento a todos los niños con cáncer y a sus padres que aceptaron participar en esta investigación.

\section{Bibliografía}

1. Caribé-Gomes F, Chimenos-Küstner E, López-López J, Finestres-Zubeldia F, Guix-Melcior B. Manejo odontológico de las complicaciones de la radioterapia y quimioterapia en el cáncer oral. Med Oral. 2003;8:178-87.

2. Peterson DE, Bensadoun RJ, Roila F. Management of oral and gastrointestinal mucositis: ESMO Clinical Practice Guidelines. Ann Oncol. 2011;22:vi78-vi84.

3. Sonis ST, Elting LS, Keefe D, Peterson DE, Schubert M, Hauer-Jensen M, et al. Perspectives on cancer therapy-induced mucosal injury. Cancer. 2004;100:1995-2025.

4. Ruiz-Esquide G, Nervi B, Vargas A, Maíz A. Tratamiento y prevención de la mucositis oral asociada al tratamiento del cáncer. Rev Med Chile. 2011;139:373-81.

5. Otmani N, Alami R, Hessissen L, Mokhtari A, Soulaymani A, Khattab M. Determinants of severe oral mucositis in paediatric cancer patients: a prospective study. Int J Paediatr Dent. 2011;21:210-6.

6. Cheng KK. Oral mucositis and quality of life of Hong Kong Chinese patients with cancer therapy. Eur J Oncol Nurs. 2007;11:36-42.

7. Soares AF, Aquino AR, Carvalho $\mathrm{CH}$, Nonaka CF, Almeida D, Pinto LP. Frequency of oral mucositis and microbiological analysis in children with acute lymphoblastic leukemia treated with $0.12 \%$ chlorhexidine gluconate. Braz Dent J. 2011;22:312-6. 


\section{R. Gutiérrez-Vargas, et al.: Instrumento para medir la calidad de vida en niños}

8. Hernández FA, Oñate SRE, Cabrerizo MMC, De- Arriba-de-la-Fuente F, Heras FI, García VV. Influence of oral health on mucositis in patients undergoing hematopoietic progenitor cell transplantation (HPCT). Med Oral Patol Oral Cir Bucal. 2012;17:e94-101.

9. Raber-Durlacher JE, Barasch A, Peterson DE, Lalla RV, Schubert MM, Fibbe WE. Oral complications and management considerations in patients treated with high-dose chemotherapy. Support Cancer Ther. 2004; 1:219-29.

10. Sonis ST, Oster G, Fuchs H, Bellm L, Bradford WZ, Edelsberg J, et al. Oral mucositis and the clinical and economic outcomes of hematopoietic stem-cell transplantation. J Clin Oncol. 2001:19:2201-5.

11. Cheng KK, Goggins WB, Lee VW, Thompson DR. Risk factors for oral mucositis in children undergoing chemotherapy: a matched case-control study. Oral Oncol. 2008;44:1019-25.

12. Joshi VK. Dental treatment planning and management for the mouth cancer patient. Oral Oncol. 2010;46:475-9.

13. Cheng KKF, Lee V, Li CH, Goggins W, Thompson D, Yuen HL, et al. Incidence and risk factors of oral mucositis in paediatric and adolescent patients undergoing chemotherapy. Oral Oncol. 2011;47:153-62.

14. Sitaresmi MN, Mostert S, Gundy CM, Sutaryo, Veerman AJ. Health-related quality of life assessment in Indonesian childhood acute lymphoblastic leukemia. Health Qual Life Outcomes. 2008;6:96-102.

15. Velarde-Jurado E, Avila-Figueroa C. Consideraciones metodológicas para evaluar la calidad de vida. Salud Publica Mex. 2002;44:448-63.

16. Ramada-Rodilla JM, Serra-Pujadas C, Delclós-Clanchet GL. Adaptación cultural y validación de cuestionarios de salud: revisión y recomendaciones metodológicas. Salud Publica Mex. 2013:55:57-66.

17. Rosenthal DI, Mendoza TR, Chambers MS, Burkett VS, Garden AS Hessell AC, et al. The M. D. Anderson symptom inventory-head and neck module, a patient-reported outcome instrument, accurately predicts the severity of radiation-induced mucositis. Int J Radiation Oncology Bio Phys. 2008;72:1355-61.

18. Cheng KKF, Leung SF, Thompson DR, Tai JW, Liang RH, Kan AS, et al. New measure of health-related quality of life for patients with oropharyngeal mucositis development and preliminary psychometric evaluation. Cancer. 2007; 109:2590-9

19. Cheng KKF, Leung SF, Liang RH, Tai JW, Yeung RM, Thompson DR A patient-reported outcome instrument to assess the impact of oropharyngeal mucositis on health-related quality of life: a longitudinal psychometric evaluation. Support Care Cancer. 2009;17:389-98.
20. Lopez CF, Oñate SR, Rodal C, Cabrerizo M. Measurement of secondary mucositis to oncohematologic treatment by means of different scale. Review. Med Oral Patol Oral Cir Bucal. 2005;10:412-21.

21. Sung $L$, Tomlinson GA, Greenberg ML, Koren G, Judd P, Ota $S$, et al. Validation of the oral mucositis assessment scale in pediatric cancer. Pediatr Blood Cancer. 2007;49:149-53.

22. Varni JW, Burwinkle TM, Katz ER, Meeske K, Dickinson P. The PedsQL in pediatric cancer, reliability and validity of the Pediatric Quality of Life Inventory Generic Core Scales, Multidimensional Fatigue Scale, and Cancer Module. Cancer. 2002;94:2090-106.

23. Magnusson D. Teoría de los tests. México: Trillas;1998.

24. Fabrigar LR, Duane WT, MacCallum RC, Strahan EJ. Evaluating the use of exploratory factor analysis in psychological research. Psychol Methods. 1999;4:271-99.

25. Nunnally JC. Teoría psicométrica. México: Trillas; 1995.

26. Bardellini E, Amadori F, Majorana A. Oral hygiene grade and quality of life in children with chemotherapy-related oral mucositis: a randomized study on the impact of a fluoride toothpaste with salivary enzymes, essential oils, proteins and colostrum extract versus a fluoride toothpaste without menthol. Int J Dent Hyg. 2016;14:314-9.

27. Bezinelli LM, Eduardo FP, Neves VD, Correa L, Lopes RM, Michel-Crosato $E$, et al. Quality of life related to oral mucositis of patients undergoing haematopoietic stem cell transplantation and receiving specialised oral care with low-level laser therapy: a prospective observational study. Eur J Cancer Care (Engl). 2016;25:668-74.

28. Gautam PA, Fernandes DJ, Vidyasagar MS, Maiya AG, Nigudgi S. Effect of low-level laser therapy on patient reported measures of oral mucositis and quality of life in head and neck cancer patients receiving chemoradiotherapy-a randomized controlled trial. Support Care Cancer. 2013;21:1421-8.

29. Lima AG, Antequera R, Peres MP, Snitcosky IM, Federico MH, Villar RC. Efficacy of low-level laser therapy and aluminum hydroxide in patients with chemotherapy and radiotherapy-induced oral mucositis. Braz Dent J. 2010;21:186-92.

30. Kushner JA, Lawrenc HP, Shoval I, Kiss TL, Devins GM, Lee L, et al. Development and Validation of a Patient-Reported Oral Mucositis Symptom (PROMS) Scale. J Can Dent Assoc. 2008;74:59.

31. Epstein JB, Robertson M, Emerton S, Phillips N, Stevenson-Moore P. Quality of life and oral function in patients treated with radiation therapy for head and neck cancer. Head Neck. 2001;23:389-98. 